\title{
Toward Optimal Resource Scheduling for Internet of Things under Imperfect CSI
}

\author{
Libo Jiao, Yulei Wu, Jiaqing Dong, and Zexun Jiang*
}

\begin{abstract}
The Internet of Things (IoT) increases the number of connected devices and supports ever-growing complexity of applications. Owing to the constrained physical size, the IoT devices can significantly enhance computation capacity by offloading computation-intensive tasks to the resource-rich edge servers deployed at the base station (BS) via wireless networks. However, how to achieve optimal resource scheduling remains a challenge due to stochastic task arrivals, time-varying wireless channels and imperfect estimation of channel state information (CSI). In this paper, by virtue of the Lyapunov optimization technique, we propose the toward optimal resource scheduling algorithm under imperfect CSI (TORS) to optimize resource scheduling in an IoT environment. A convex transmit power and subchannel allocation problem in TORS is formulated. This problem is then solved via the Lagrangian dual decomposition method. We derive analytical bounds for the time-averaged system throughput and queue backlog. We show that TORS can arbitrarily approach the optimal system throughput by simply tuning an introduced control parameter $\beta$ without prior knowledge of stochastic task arrivals and the CSI of wireless channels. Extensive simulation results confirm the theoretical analysis on the performance of TORS.
\end{abstract}

Index Terms-Resource scheduling, imperfect CSI, channel estimation, Lyapunov optimization.

\section{INTRODUCTION}

$\mathbf{I}$ $\mathrm{N}$ the coming era of Internet of Things (IoT), an increasing number of IoT devices, ranging from RFIDs to biological sensors, are connected to the base station (BS) through wireless networks. Due to the limited computing resources and battery capacities, most IoT devices cannot afford the sophisticated IoT applications which are computation-intensive and time-sensitive. The performance of IoT devices can be boosted by offloading the complex tasks to a capable edge server, which is located at the BS and has high computation capability and large storage capacity. This is so-called mobile edge computing (MEC). By utilizing the resources and services offered by edge cloud, MEC has obtained the potential benefits (e.g., low energy consumption, low latency and high reliability, etc.), which are of particular interest to the future IoT community [1]-[4].

To accommodate the ever-increasing volume of mobile traffic and offload the overloaded traffic, orthogonal frequency division multiplexing access (OFDMA) technology has been

L. Jiao, J. Dong, and Z. Jiang are with the Department of Computer Science and Technology, Tsinghua University, Beijing, 100084 China. E-mail: (jlb15@mails.tsinghua.edu.cn,djq13@mails.tsinghua.edu.cn, and jiangzx14@mails.tsinghua.edu.cn).

$\mathrm{Y}$. Wu is with the College of Engineering, Mathematics and Physical Sciences, University of Exeter, Exeter EX4 4QF, U.K. E-mail: y.1.wu@exeter.ac.uk.

*Corresponding author: Zexun Jiang. widely used in modern and envisaged cellular standards such as WiMAX and 3GPP LTE [5][6]. Extensive research works have been devoted to maximize the overall throughput of OFDMA-based systems by devising appropriate resource scheduling policies [7]-[12], such as power allocation, subchannel assignment, mode selection and BS operation. All these research works are studied on the assumption that the perfect channel state information (CSI) can be obtained. However, due to channel estimation error, the transmitting nodes only have imperfect CSI in practical networks. Moreover, optimal resource scheduling has also been hindered by the stochastic and complex nature of real-world networking environment, including time-varying wireless channels and stochastic task arrivals. We therefore need to take the stochasticity and unpredictability into consideration and optimize resource scheduling to maximize the system throughput of IoT networks.

The resource scheduling problem under the imperfect CSI for OFDMA systems has drawn tremendous research interests [13]-[19]. A power allocation scheme was proposed in [13] to maximize the energy efficiency of small cells for downlink non-orthogonal multiple access (NOMA) heterogeneous networks based on imperfect CSI. The authors in [14] proposed an optimal power allocation solution to maximize the energy efficiency in NOMA systems with imperfect CSI and user quality of service constraint. A new optimization framework was developed in [15] for jointly optimizing power allocation, user selection and precoding in multi-cell multi-user multipleinput multiple-output (MU-MIMO) systems with imperfect CSI. Bai et al. [16] studied the fair resource allocation of device-to-device (D2D) communication underlaying cellular systems with CSI estimation error; they developed a joint resource scheduling scheme about resource chunk and power allocation among the D2D and cellular network users. Liu et al. [17] proposed an optimal power allocation scheme for the downlink multi-user NOMA beamforming system with imperfect CSI. However, these works [13]-[17] do not consider the stochastic packet arrival characteristics and network stability (i.e., poor channel situation and/or low transmit power may lead to network congestion). Liu et al. [18] proposed a joint power and subchannel allocation policy for the downlink of an OFDMA system, with various practical considerations including stochastic packet arrivals, time-varying channels, and imperfect CSI, but the objective of their work was to minimize the total power consumption. In [19], a resource allocation scheme was developed to maximize a network utility, but they ignored the factors of IoT device finite buffer size and subchannel dynamic assignment policy. 
In this paper, we propose a joint traffic admission control, transmit power allocation and subchannel assignment mechanism to maximize the overall system throughput, while considering CSI estimation error and the constraints of IoT device buffer size and network stability. Specifically, we employ a stochastic optimization model to cope with evolving network environment. We aim at maximizing the long term time-averaged system throughput while guaranteeing queue stability to avoid network congestion. The main contributions of this work are summarized as follows:

1) The resource scheduling problem is formulated as a stochastic optimization model to maximize the long term time-averaged system throughput subject to network stability constraint.

2) Based on our model, we develop a general and effective algorithm, referred as the toward optimal resource scheduling algorithm under imperfect CSI (TORS). By exploiting Lyapunov optimization technique, this algorithm performs resource scheduling without any prior knowledge of network state information, while at the same time guaranteeing network stability.

3) We theoretically analyze the performance of the proposed TORS algorithm and prove the network stability and system throughput performance. Extensive simulation results are conducted to verify the theoretical analysis and the performance of TORS. The evaluation conforms that our solution can flexibly tune the time-averaged system throughput and data queue length by simply adjusting an introduced control parameter $\beta$.

Compared to our previous work [19], the main differences are as follows: (1) Owing to the finite buffer size of IoT devices, we employ the virtual admission queue technique and a carefully designed Lyapunov function to avoid buffer overflow. (2) We build a more general resource scheduling model which combines the power allocation and subchannel alignment. (3) We conduct extensive simulation results to validate the effectiveness of the proposed TORS algorithm.

The remainder of this paper is organized as follows. Section II provides an overview of the system model followed by the problem formulation in Section III. The TORS algorithm is developed in Section IV. Section V carries out the performance analysis of the proposed TORS algorithm. Simulation results and analysis are presented in Section VI. Finally, we conclude our paper in Section VII.

\section{THE SySTEM MOdEL}

As shown in Fig 1, we consider a typical IoT system, consisting of a BS and $M$ IoT devices (e.g., smart TVs, smart speakers, wearables and smart cameras). An edge server deployed at BS has computation capacity to process the sensory data offloaded from the IoT devices through wireless channels.

\section{A. Uplink Traffic Admission Control}

We assume that the IoT system operates in a time-slotted manner with time slot index $t \in\{0,1,2, \ldots\}$. The sensory data generated by IoT devices arrives randomly every time

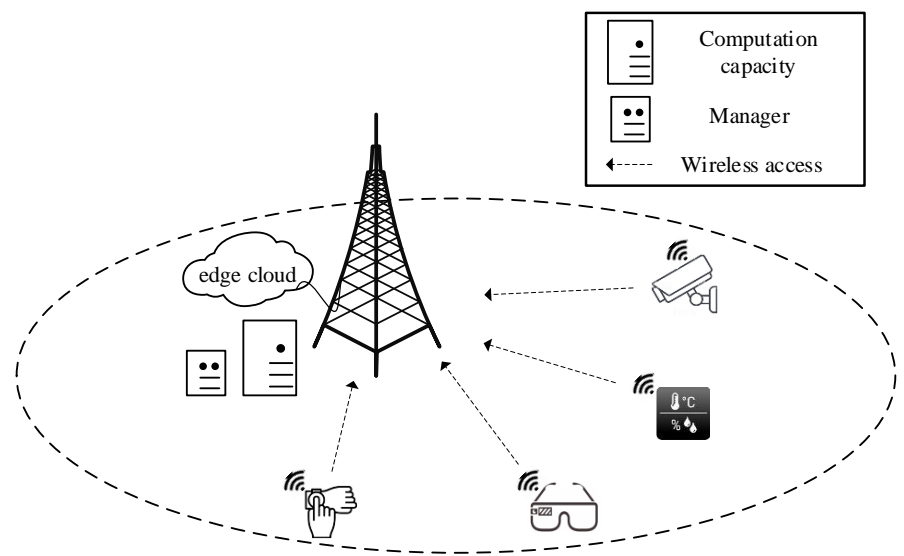

Fig. 1. An illustration of IoT systems

slot. Each device maintains a buffer to temporally store the arrived data until they are transmitted to the edge cloud for further processing. We introduce $\boldsymbol{A}(t)=\left\{A_{m}(t)\right\}$ to denote the random process of data arrivals with the maximum $A_{\max }$, where $A_{m}(t)$ is the amount of new sensory data that arrives at the IoT device $m$ at time slot $t$. Assume that $\boldsymbol{A}(t)$ is an independently and identically distributed (i.i.d.) random process with arrival rate $\boldsymbol{\lambda}=\left\{\lambda_{m}\right\}$, where $\lambda_{m}$ is the timeaveraged sensory data arrival rate of the IoT device $m$.

To avoid congestion under massive sensory data or continuous poor network condition, we employ an admission control strategy to adjust the traffic rate that the amount of sensory data can be admitted to the device buffer at each time slot. The admission control scheme is involved with adjusting the admission rate, denoted by $\boldsymbol{R}(t)=\left\{R_{m}(t)\right\}$, where $R_{m}(t)$ is the admission rate of the device $m$ at time slot $t$, and it should satisfy the following constraint

$$
R_{m}(t) \leq A_{m}(t) \leq A_{\max }, \forall m, t
$$

Obviously, for any IoT device, the amount of admission data must be less than or equal to the amount of generated sensory data at each time slot.

\section{B. Communication Model}

There are $N$ number of subchannels in the system, each with a bandwidth of $F$. Let $\boldsymbol{D}(t)=\left\{d_{m n}(t)\right\}$ be the binary subchannel allocation indicator matrix, where $d_{m n}(t)=1$ denotes that the $n$th subchannel is assigned to the IoT device $m$ at time slot $t$, and $d_{m n}(t)=0$ otherwise. Each subchannel $n$ can be allocated to at most one IoT device in each time slot. Thus, we have the following constraint

$$
\sum_{m=1}^{M} d_{m n}(t) \leq 1, \forall n, t
$$

To obtain the channel transmission rate, we need to know the perfect CSI at the BS, i.e., the exact value of channel gain. Nevertheless, it is subject to channel uncertainty in practice. With the aid of pilot signals transmitted from its corresponding IoT devices, BS can estimate the CSI, e.g., background noise and channel gains [20]. To acquire the CSI, we employ 


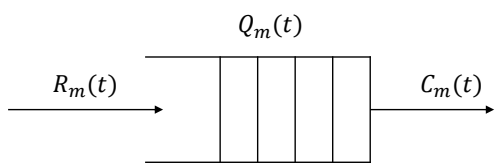

Fig. 2. The arrival and departure processes

the minimum mean squared error (MMSE) estimator as the channel estimation method. Let $h_{m n}(t)$ denote the channel gain from the transmitter IoT device $m$ to the receiver BS on a subchannel $n$ at time slot $t$. With the assumption of MMSE channel estimator, the estimation and estimation error of CSI, denoted by $\hat{h}_{m n}(t)$ and $\tilde{h}_{m n}(t)$, are uncorrelated Gaussian random variables, with zero means and variance $\hat{\sigma}^{2}=\frac{\phi_{t n}}{1+\phi_{t n}}$ and $\tilde{\sigma}^{2}=\frac{1}{1+\phi_{t n}}$, respectively. $\phi_{t n}$ denotes the signal-to-noiseratio (SNR) of pilot transmission [21]. The relationship among them is described as follows [18] [22]

$$
h_{m n}(t)=\hat{h}_{m n}(t)+\tilde{h}_{m n}(t)
$$

Assuming that the IoT system adopts a continuous rate policy, then we employ the Shannon capacity formula to describe the transmission rate. Specifically, the uplink transmission rate of the IoT device $m$ on the subchannel $n$ at time slot $t$, denoted by $C_{m n}(t)$, can be computed as

$$
C_{m n}(t)=F \log _{2}\left(1+\frac{\phi_{m n}(t) g_{m n}(t)}{\phi_{m n}(t) \tilde{\sigma}^{2}+1}\right)
$$

where $\phi_{m n}(t)=p_{m n}(t) / w \cdot p_{m n}(t)$ denotes the transmit power allocated to the device $m$ on the subchannel $n$ at time slot $t . w$ is the power of additive white Gaussian noise per subchannel, and $g_{m n}(t)=\left|\hat{h}_{m n}(t)\right|^{2}$.

\section{Queueing Model and System Dynamics}

Let $\boldsymbol{Q}(t)=\left\{Q_{m}(t)\right\}$ denote the data queue backlog of all IoT devices at time slot $t$, where $Q_{m}(t)$ denotes the queue backlog of the device $m$ at time slot $t$, and it is updated along the time, as given by

$$
Q_{m}(t+1)=\max \left[Q_{m}(t)-C_{m}(t), 0\right]+R_{m}(t)
$$

where $C_{m}(t)=\sum_{n=1}^{N} d_{m n}(t) C_{m n}(t)$ is the total transmission capacity of the device $m$ at time slot $t$. It also denotes the serving rate of data queue on the device $m . R_{m}(t)$ implies the arrival rate for the data queue on the device $m$ at time slot $t$. Finally, both the serving rate $C_{m}(t)$ and the arrival rate $R_{m}(t)$ have major implications for the future queue backlog $Q_{m}(t+1)$, which is shown in Fig. 2 .

A discrete time queue $Z(t)$ is defined as strongly stable if the following condition holds

$$
\lim _{T \rightarrow \infty} \frac{1}{T} \sum_{t=0}^{T-1} \mathbb{E}\{|Z(t)|\}<\infty
$$

In real systems, we call the queue is strongly stable if the long term time-averaged arrival rate injected into the queue is smaller than or equal to the long term time-averaged departure rate from the queue. Then, network congestion can be avoided
TABLE I

THE IMPORTANT PARAMETERS AND NOTATIONS

\begin{tabular}{|c|l|}
\hline Symbol & \multicolumn{1}{c|}{ Meaning } \\
\hline$M$ & Number of IoT devices \\
\hline$N$ & Number of subchannels \\
\hline$F$ & Bandwidth of each subchannel \\
\hline$h_{m n}(t)$ & $\begin{array}{l}\text { Channel gains on subchannel } n \text { from device } m \text { to BS } \\
\text { at time slot } t\end{array}$ \\
\hline$\hat{h}_{m n}(t)$ & $\begin{array}{l}\text { Channel estimation on subchannel } n \text { from device } m \text { to BS } \\
\text { at time slot } t\end{array}$ \\
\hline$\tilde{h}_{m n}(t)$ & $\begin{array}{l}\text { Channel estimation error on subchannel } n \text { from BS } \\
\text { to device } m \text { at time slot } t\end{array}$ \\
\hline$d_{m n}(t)$ & Binary subchannel allocation indicator \\
\hline$C_{m n}(t)$ & $\begin{array}{l}\text { Transmission rate for device } m \text { on subchannel } n \text { at time } \\
\text { slot } t\end{array}$ \\
\hline$p_{m n}(t)$ & $\begin{array}{l}\text { Transmit power allocated on subchannel } n \text { to device } m \text { at } \\
\text { time slot } t\end{array}$ \\
\hline$R_{m}(t)$ & Admission rate of device $m$ at time slot $t$ \\
\hline$Q_{m}(t)$ & Queue backlog at device $m$ at time slot $t$ \\
\hline$A_{m}(t)$ & Amount of arrived data at device $m$ at time slot $t$ \\
\hline$X_{m}(t)$ & Virtual admission rate for device $m$ at time slot $t$ \\
\hline$\beta$ & Lyapunov control parameter \\
\hline $\boldsymbol{S}(t)$ & System state at time slot $t$ \\
\hline \hline
\end{tabular}

that all data placed into the queue will be transmitted at last if the queue is strongly stable.

\section{Problem Formulation}

To improve system throughput, we aim at maximizing the long term time-averaged admission rate while satisfying network stability constraint. Then, the resource scheduling is formulated as the following stochastic optimization problem

$$
\begin{array}{ll}
\text { P1: } \max \lim _{T \rightarrow \infty} \frac{1}{T} \sum_{t=0}^{T-1} R_{\text {sum }}(t) \\
\text { s.t. C1: } R_{m}(t) \leq A_{m}(t) \leq A_{\max }, \forall m, t \\
\text { C2: } p_{m n}(t) \geq 0, \forall m, n, t \\
\text { C3: } \sum_{m=1}^{M} \sum_{n=1}^{N} p_{m n}(t) \leq P_{\max }, \forall t \\
\text { C4: } d_{m n}(t) \in\{0,1\}, \forall m, n, t \\
\text { C5: } \sum_{m=1}^{M} d_{m n}(t) \leq 1, \forall n, t \\
\text { C6: Queues } Q(t) \text { are strongly stable. }
\end{array}
$$

where $R_{\text {sum }}(t)=\sum_{m=1}^{M} R_{m}(t)$ denotes the overall system throughput achieved by all IoT devices. $\mathrm{C} 1$ is the admission control constraint to ensure the account of admitted data cannot exceed the amount of newly arrived data at each time slot. C2 is a nonnegative power allocation constraint. C3 is the peak transmit power constraint. $\mathrm{C} 4$ and $\mathrm{C} 5$ indicate that each subchannel is exclusively allocated among devices. C6 is the network stability constraint. 


\section{Online Resource Scheduling Algorithm}

In this section, we propose an online resource scheduling algorithm TORS to solve the problem P1. Considering that there is a finite buffer size of each device, we introduce the virtual admission queue to assist in designing our algorithm.

\section{A. The Virtual Admission Queue}

In practical IoT systems, the buffer size, denoted by $b_{\max }$, is finite. Similar with the previous work [7], we employ virtual admission rate queues $\boldsymbol{X}(t)=\left\{X_{m}(t)\right\}$ to avoid buffer overflow. It is updated along the time as

$$
X_{m}(t+1)=\max \left[X_{m}(t)-R_{m}(t), 0\right]+V_{m}(t)
$$

where $\boldsymbol{V}(t)=\left\{V_{m}(t)\right\}$ is the virtual admission rate of data queues $\boldsymbol{Q}(t)$.

By introducing virtual admission rate, the original stochastic optimization problem P1 can be reformulated as

$$
\begin{aligned}
& \text { P2: } \max \lim _{T \rightarrow \infty} \frac{1}{T} \sum_{t=0}^{T-1} R_{\text {sum }}(t) \\
& \text { s.t. C1, C2, C3, C4, C5, and C6 } \\
& \text { C7: Queues } \boldsymbol{X}(t) \text { are strongly stable. }
\end{aligned}
$$

\section{B. Online Algorithm for Optimal Resource Scheduling}

Let $\boldsymbol{\Theta}(t)=[\boldsymbol{Q}(t), \boldsymbol{X}(t)]$ be the vector of all real and virtual queue backlogs employed in the IoT system. We introduce $L$, which is widely used to guarantee queue stability in the Lyapunov function [23][24], for the constraints C6 and C7. The perturbed Lyapunov function can be constructed as

$$
\begin{aligned}
L(\boldsymbol{\Theta}(t))= & \frac{1}{2} \sum_{m=1}^{M} \frac{1}{b_{\max }} X_{m}(t) Q_{m}(t)^{2} \\
& +\frac{1}{2} \sum_{m=1}^{M} \frac{b_{\max }-A_{\max }}{b_{\max }} X_{m}(t)^{2}
\end{aligned}
$$

Without loss of generality, we assume $L(\Theta(0))=0$ that all queues length are zero when $t=0$. The one-slot conditional Lyapunov drift is defined as the conditional expectation of the difference of the Lyapunov function between two consecutive slots, and it is defined as

$$
\Delta(\boldsymbol{\Theta}(t))=\mathbb{E}\{L(\boldsymbol{\Theta}(t+1))-L(\boldsymbol{\Theta}(t)) \mid \boldsymbol{\Theta}(t)\}
$$

In addition, the Lyapunov drift-minus-reward term is defined as

$$
\Delta(t)=\Delta(\boldsymbol{\Theta}(t))-\beta \mathbb{E}\left\{V_{\text {sum }}(t) \mid \Theta(t)\right\}
$$

where $V_{\text {sum }}(t)=\sum_{m=1}^{M} V_{m}(t)$ and $\beta$ is a nonnegative tunable parameter. Lyapunov optimization theory [25][26] guides us to minimize (12), such that the time-averaged system throughput approaches to the optimal value, whereas network queue backlogs are pushed towards a lower congestion state. Based on the Lyapunov optimization theory, the upper bound of (12) is given in the following theorem.
Theorem 1(Upper Bound): For any queue backlogs and actions, $\Delta(t)$ is upper bounded by

$$
\begin{aligned}
& \Delta(\boldsymbol{\Theta}(t))-\beta \mathbb{E}\left\{V_{\text {sum }}(t) \mid \boldsymbol{\Theta}(t)\right\} \\
& \leq B+\sum_{m=1}^{M} \mathbb{E}\left\{\frac{C_{m}(t)^{2}+R_{m}(t)^{2}}{2 b_{\max }} X_{m}(t) \mid \boldsymbol{\Theta}(t)\right\} \\
& -\sum_{m=1}^{M} \mathbb{E}\left\{\frac{X_{m}(t) Q_{m}(t)}{b_{\max }} C_{m}(t) \mid \boldsymbol{\Theta}(t)\right\} \\
& +\sum_{m=1}^{M} \mathbb{E}\left\{R_{m}(t) \frac{X_{m}(t)}{b_{\max }}\left[Q_{m}(t)-\left(b_{\max }-A_{\max }\right)\right] \mid \boldsymbol{\Theta}(t)\right\} \\
& +\sum_{m=1}^{M} \mathbb{E}\left\{V_{m}(t)\left(\frac{b_{\max }-A_{\max }}{b_{\max }} X_{m}(t)-\beta\right) \mid \boldsymbol{\Theta}(t)\right\}
\end{aligned}
$$

where $B$ is a positive constant, which satisfies the following constraint for all $t$.

$$
\begin{aligned}
B \geq B(t)= & \frac{b_{\max }-A_{\max }}{2 b_{\max }} \sum_{m=1}^{M}\left(R_{m}(t)^{2}+V_{m}(t)^{2}\right) \\
& +\frac{M A_{\max } b_{\max }}{2}
\end{aligned}
$$

Proof: See Appendix A.

Based on the stochastic optimization theory in [25], we need to minimize the upper bound of the drift-minus-reward term with the same constraints except for the network stability. Back to our case, it is required to minimize the right-hand side (RHS) of (13) at each time slot $t$ to solve the problem P1 subject to C1-C5, and C6 and C7 are the network stability constraints. Then, we have transformed the original stochastic optimization problem P1 into a series of successive instantaneous static optimization problems.

\section{Algorithm Design}

The complexity of the problem P2 comes from the joint optimization of admission control and resource scheduling operation. One method to reduce the complexity is to decompose the joint problem into admission control subproblem and resource scheduling subproblem, and then the two subproblems can be solved separately. By analyzing the structure of the problem, we divide it into two separate parts. First, an admission subproblem determines how much data to admit to the data queue based on a threshold criterion. Then, a resource scheduling subproblem finds the optimal transmit power allocation and subchannel assignment scheme based on current system state by using the Lagrangian dual decomposition method. The details of the procedure to solve the problem $\mathrm{P} 2$ is summarized in Algorithm 1, referred to as the TORS in the paper.

1) Admission Control Decision: By observing that the fourth term on the RHS of (13) involves the admission control decision $R_{m}(t)$, we divide it into $M$ subproblems as

$$
\begin{aligned}
& \min _{R_{m}(t)} R_{m}(t) \frac{X_{m}(t)}{b_{\max }}\left[Q_{m}(t)-\left(b_{\max }-A_{\max }\right)\right] \\
& \text { s.t. C1. }
\end{aligned}
$$




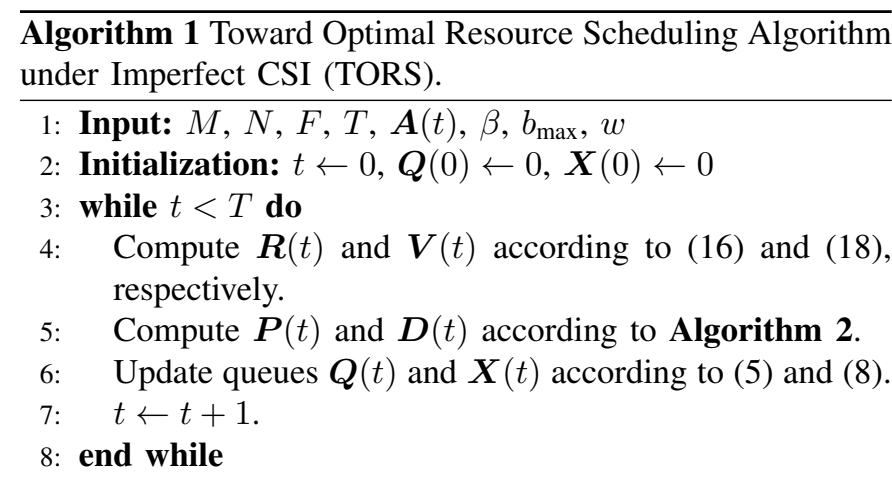

The corresponding solution to (15) is

$$
R_{m}(t)= \begin{cases}A_{m}(t), & \text { if } Q_{m}(t) \leq b_{\max }-A_{\max } \\ 0, & \text { otherwise. }\end{cases}
$$

Equation (16) denotes that the IoT device $m$ will accept all arrival data to data queue if its queue backlog $Q_{m}(t)$ is smaller than the threshold $\left(b_{\max }-A_{\max }\right)$ at time $t$. Otherwise, it would reject all new arrival data.

We observe that the fifth term on the RHS of (13) involves the virtual admission decision $V_{m}(t)$. Similarly, we divide it into $M$ subproblems as

$$
\min _{V_{m}(t)} V_{m}(t)\left(\frac{b_{\max }-A_{\max }}{b_{\max }} X_{m}(t)-\beta\right)
$$

The corresponding solution to (17) is

$$
V_{m}(t)= \begin{cases}A_{m}(t), & \text { if }\left(b_{\max }-A_{\max }\right) X_{m}(t) / b_{\max } \leq \beta \\ 0, & \text { otherwise. }\end{cases}
$$

2) Resource Scheduling Decision: Observe that the third term on the RHS of (13) involves the transmit power and subchannel indicator variables. We reformulate these terms as

$$
\begin{gathered}
\max _{\boldsymbol{P}(t), \boldsymbol{D}(t)} \sum_{m=1}^{M} \sum_{n=1}^{N} \frac{X_{m}(t) Q_{m}(t)}{b_{\max }} d_{m n}(t) C_{m n}(t) \\
\text { s.t. C2, C3, C4, and C5. }
\end{gathered}
$$

where $\boldsymbol{P}(t)=\left\{p_{m n}(t)\right\}$ and $\boldsymbol{D}(t)=\left\{d_{m n}(t)\right\}$.

This optimization problem formulation is a typical mixedinteger non-convex programming problem. The optimal solution of (19) can be acquired through branch and bound methods, but the optimality of solutions at each time slot has a high computational complexity. To overcome the complexity limitation of integer variables, we extend the constraint $\mathrm{C} 4$ with the relaxation. This relaxation is indicated by replacing discrete variable $d_{m n}(t)$ with continuous real variable $\bar{d}_{m n}(t)$, where $0 \leq \bar{d}_{m n}(t) \leq 1$. We introduce the auxiliary variable $\bar{p}_{m n}(t)=\bar{d}_{m n}(t) p_{m n}(t)$ for the device $m$ on the subchannel $n$ during time slot $t$. With the help of relaxation and auxiliary variable, the problem (19) can be reformulated into

$$
\begin{aligned}
\max _{\overline{\boldsymbol{P}}(t), \overline{\boldsymbol{D}}(t)} & \sum_{m=1}^{M} \sum_{n=1}^{N} \frac{F X_{m}(t) Q_{m}(t)}{b_{\max }} \bar{d}_{m n}(t) \\
& \times \log _{2}\left(1+\frac{\bar{p}_{m n}(t) g_{m n}(t)}{\bar{p}_{m n}(t) \tilde{\sigma}^{2}+w}\right) \\
\text { s.t. } \mathrm{C} 8: \sum_{m=1}^{M} \sum_{n=1}^{N} \bar{p}_{m n}(t) \leq P_{\max }, \forall t & \text { C9: } \bar{p}_{m n}(t) \geq 0, \forall m, n, t \\
& \text { C10: } \bar{d}_{m n}(t) \in[0,1], \forall m, n, t \\
& \text { C11: } \sum_{m=1}^{M} \bar{d}_{m n}(t) \leq 1, \forall n, t
\end{aligned}
$$

where $\overline{\boldsymbol{P}}(t)=\left\{\bar{p}_{m n}(t)\right\}$ and $\overline{\boldsymbol{D}}(t)=\left\{\bar{d}_{m n}(t)\right\}$. Considering that the constraints $\mathrm{C} 8, \mathrm{C} 9, \mathrm{C} 10$, and $\mathrm{C} 11$ are linear and the objective function is jointly concave with respective to $\bar{p}_{m n}(t)$ and $\bar{d}_{m n}(t)$. Thus, the problem in (20) is convex optimization problem [27], which can be solved by the Lagrangian dual decomposition method. The Lagrangian function is formulated as

$$
\begin{aligned}
& L(\overline{\boldsymbol{P}}(t), \overline{\boldsymbol{D}}(t), \mu) \\
& =\sum_{m=1}^{M} \sum_{n=1}^{N} \frac{F X_{m}(t) Q_{m}(t)}{b_{\max }} \bar{d}_{m n}(t) \\
& \quad \times \log _{2}\left(1+\frac{\bar{p}_{m n}(t) g_{m n}(t)}{\bar{p}_{m n}(t) \tilde{\sigma}^{2}+w}\right) \\
& \quad-\mu\left(\sum_{m=1}^{M} \sum_{n=1}^{N} \bar{p}_{m n}(t)-P_{\max }\right)
\end{aligned}
$$

where $\mu$ is the Lagrange multipliers for the constraint C8. The Lagrangian dual function of the problem is defined as

$$
y(\mu)=\max _{\overline{\boldsymbol{P}}(t), \overline{\boldsymbol{D}}(t)} L(\overline{\boldsymbol{P}}(t), \overline{\boldsymbol{D}}(t), \mu)
$$

The dual problem can be given as

$$
\begin{aligned}
& \min _{\mu} y(\mu) \\
& \text { s.t. } \mu \geq 0
\end{aligned}
$$

The Lagrangian dual function in (22) can be decomposed into $M \times N$ subproblems. Accordingly, the Lagrangian function is rewritten as

$$
\begin{aligned}
& L(\overline{\boldsymbol{P}}(t), \overline{\boldsymbol{D}}(t), \mu) \\
& =\sum_{m=1}^{M} \sum_{n=1}^{N} L_{m n}(\overline{\boldsymbol{P}}(t), \overline{\boldsymbol{D}}(t), \mu)+\mu P_{\max }
\end{aligned}
$$

$$
p_{m n}^{*}(t)= \begin{cases}\frac{F X_{m}(t) Q_{m}(t)}{b_{\max } \mu \ln 2}-\frac{w}{g_{m n}(t)}, & \tilde{\sigma}^{2}=0 \\ \frac{w\left(2 \tilde{\sigma}^{2}+g_{m n}(t)\right)}{2 \tilde{\sigma}^{2}\left(\tilde{\sigma}^{2}+g_{m n}(t)\right)}\left(\sqrt{1+\frac{4 \tilde{\sigma}^{2}\left(\tilde{\sigma}^{2}+g_{m n}(t)\right)}{\left(2 \tilde{\sigma}^{2} w+g_{m n}(t) w\right)^{2}}\left[S_{m n}(t)\right]^{+}}-1\right), & \text { otherwise. }\end{cases}
$$

where $S_{m n}(t)=\frac{w F X_{m}(t) Q_{m}(t) g_{m n}(t)}{b_{\max } \mu \ln 2}-w^{2},[x]^{+}=\max [x, 0]$. 
where

$$
\begin{aligned}
& L_{m n}(\overline{\boldsymbol{P}}(t), \overline{\boldsymbol{D}}(t), \mu) \\
& =\frac{F X_{m}(t) Q_{m}(t)}{b_{\max }} \bar{d}_{m n}(t) \log _{2}\left(1+\frac{\bar{p}_{m n}(t) g_{m n}(t)}{\bar{p}_{m n}(t) \tilde{\sigma}^{2}+w}\right) \\
& \quad-\mu \bar{p}_{m n}(t)
\end{aligned}
$$

Then, taking the partial derivative of $L_{m n}(t)$ with respect to $\bar{p}_{m n}(t)$ yields

$$
\begin{aligned}
\frac{\partial L_{m n}(t)}{\partial \bar{p}_{m n}(t)}= & \frac{w F X_{m}(t) Q_{m}(t) \bar{d}_{m n}(t) g_{m n}(t) \log _{2} e}{b_{\max }\left[\left(\tilde{\sigma}^{2}+g_{m n}(t)\right) \bar{p}_{m n}(t)+w\right]} \\
& \times \frac{1}{\bar{p}_{m n}(t) \tilde{\sigma}^{2}+w}-\mu
\end{aligned}
$$

According to the Karush-Kuhn-Tucker conditions [27], the optimal power allocation which denoted by $\bar{p}_{m n}^{*}(t)$ must satisfy the following constraints

$$
\left\{\begin{aligned}
\frac{\partial L_{m n}(t)}{\partial \bar{p}_{m n}(t)} & =0 \\
\bar{p}_{m n}(t) & \geq 0
\end{aligned}\right.
$$

Then, by solving (28), we derive the optimal $\bar{p}_{m n}^{*}(t)=$ $p_{m n}^{*}(t) \bar{d}_{m n}(t)$, where $p_{m n}^{*}(t)$ can be given by (25).

Substituting the optimal $\bar{p}_{m n}^{*}(t)$ into (23), the Lagrangian dual function can be also rewritten as

$$
\max _{\overline{\boldsymbol{D}}(t)} \sum_{m=1}^{M} \sum_{n=1}^{N} \Upsilon_{m n}(t) \bar{d}_{m n}(t)+\mu P_{\max }
$$

where

$$
\begin{aligned}
\Upsilon_{m n}(t)= & \frac{F X_{m}(t) Q_{m}(t)}{b_{\max }} \log _{2}\left(1+\frac{p_{m n}^{*}(t) g_{m n}(t)}{p_{m n}^{*}(t) \tilde{\sigma}^{2}+w}\right) \\
& -\mu p_{m n}^{*}(t)
\end{aligned}
$$

For any subchannel $n$, it should be allocated to the device with the largest $\Upsilon_{m n}(t)$, and then the optimal subchannel assignment can be given by

$$
d_{m n}^{*}(t)= \begin{cases}1, & m=\arg \max _{u} \Upsilon_{u n}(t) \text { and } \Upsilon_{u n}(t) \geq 0 \\ 0, & \text { otherwise. }\end{cases}
$$

To address the minimization problem in (23), the subgradient method is employed to update the Lagrangian multiplier $\mu$, and it is updated as

$$
\mu^{i+1}=\left[\mu^{i}-\eta\left(P_{\max }-\sum_{m=1}^{M} \sum_{n=1}^{N} \bar{p}_{m n}^{*}(t)\right)\right]^{+}
$$

where $i$ denotes the iteration index, and $\eta$ is the step size. $I_{\max }$ is the maximum number of iterations. When the subgradient method converges, the power allocation and subchannel assignment are finished. The details of the procedure to solve power allocation and subchannel assignment are summarized in Algorithm 2.

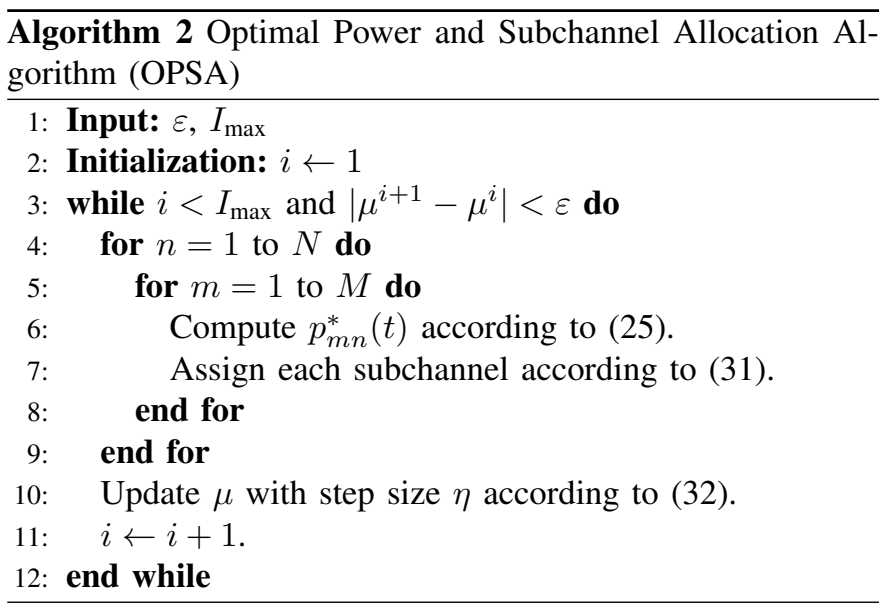

\section{Computation Complexity Analysis}

The computation complexity in each time slot is dominated by the iterative procedure from Step 4 to 11 in Algorithm 2 . The computation complexity for each iteration is $O(M N)$. The Lagrangian multiplier $\mu$ takes $O\left(1 / \varepsilon^{2}\right)$ iterations to the desired state. Thus, the total complexity of the OPSA algorithm at each time slot is $O\left(M N / \varepsilon^{2}\right)$. The OPSA algorithm with a polynomial computation complexity facilitates the practical implementation.

\section{Algorithm Performance}

Theorem 2: The performance of the TORS is given as follows.

(a) All queues $\boldsymbol{Q}(t)$ and $\boldsymbol{X}(t)$ are strongly stable, and $Q_{m}(t) \leq b_{\max }$.

(b) Given $\epsilon>0$, if $b_{\max }-A_{\max }>\left(C_{\max }^{2}+A_{\max }^{2}\right) / 2 \epsilon$, then the long term time-averaged queue backlog is bounded by

$$
\lim _{T \rightarrow \infty} \frac{1}{T} \sum_{t=0}^{T-1} \sum_{m=1}^{M} \mathbb{E}\left\{X_{m}(t)\right\} \leq \frac{B+\beta M A_{\max }}{\vartheta}
$$

(c) The long term time-averaged overall system throughput achieved by all IoT devices is bounded by

$$
\lim _{T \rightarrow \infty} \frac{1}{T} \sum_{t=0}^{T-1} \sum_{m=1}^{M} \mathbb{E}\left\{r_{m}\right\} \geq-\frac{B}{\beta}+\sum_{m=1}^{M} r_{m, \epsilon}^{*}
$$

Proof: See Appendix B.

\section{Simulation Results And Analysis}

In this section, we present simulation results to evaluate the performance of our proposed online resource scheduling algorithm. We consider an example of the uplink IoT network consisting of a BS and $M=5$ IoT devices. Particularly, the arrival rate of sensory data $A_{m}(t) \sim P\left[\lambda_{m}\right]$ Mbps and $\lambda_{m} \sim U[5,8]$, where $P\left[\lambda_{m}\right]$ denotes a Poisson distribution with average arrival rate $\lambda_{m}$, and $U[a, b]$ denotes a random uniform distribution within $[a, b]$. We assume that the number of available subchannels $N=8$, the bandwidth $F=1 \mathrm{MHz}$, the buffer size $b_{\max }=5 \lambda_{m}$ Mbits, and the power spectral 

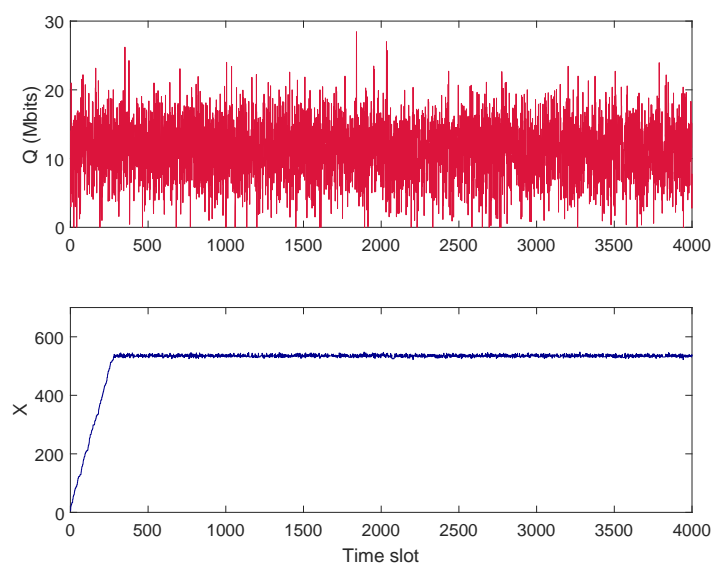

Fig. 3. Illustration of all queues stability with $\beta=250$ and $\tilde{\alpha}^{2}=0.3$.

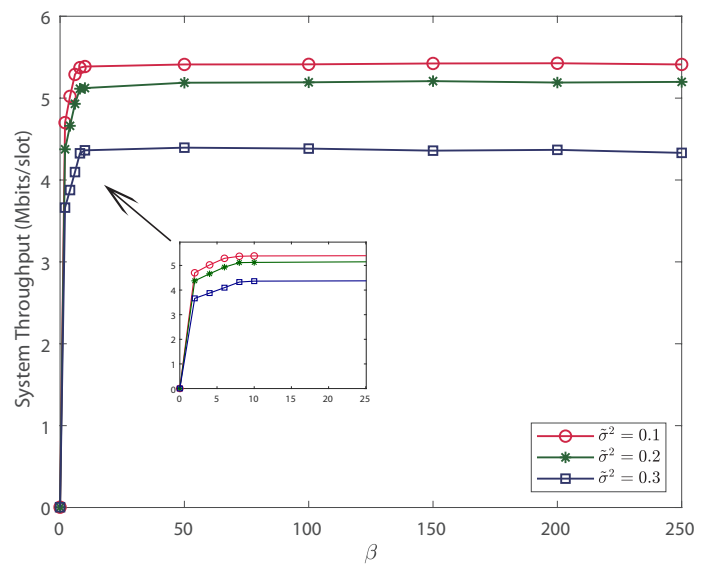

Fig. 4. The system throughput versus $\beta$.

density of noise is $10^{-8} \mathrm{~W}$. The simulation is carried out for $T=4000$ consecutive time slots.

Fig. 3 demonstrates the queue stability over different time slots with $\beta=250$ whose value does not have the impact on the queue stability from the extensive simulations. We take the queues of the device $m=1$ as an example since the data queues $\boldsymbol{Q}(t)$ and virtual admission queues $\boldsymbol{X}(t)$ follow similar trends. This figure shows the dynamics of device's data queue backlog $Q_{1}$ and virtual admission queue $X_{1}$. The arrival data rate of the device 1 is $\lambda_{1}=6 \mathrm{Mbps}$ and the buffer size is $b_{\max }=30 \mathrm{Mbits}$. It can be seen that these queues are strictly bounded and actual data queue $Q_{1}$ is strictly lower than the buffer size $b_{\max }$, which validates the conclusion of Theorem $2(a)$.

Fig. 4 plots the time-averaged system throughput of the proposed approach by varying the control parameter $\beta$. Here, $\beta \in[0,250]$ provides a reasonable dynamic range to display the impact of $\beta$ on the queue backlog. First, we observe that the system throughput keeps increasing at the beginning when $\beta \leq 10$, and finally stabilizes around the optimal system throughput level. This is due to the fact that the Lyapunov drift-minus-reward function in the proposed algorithm is min-

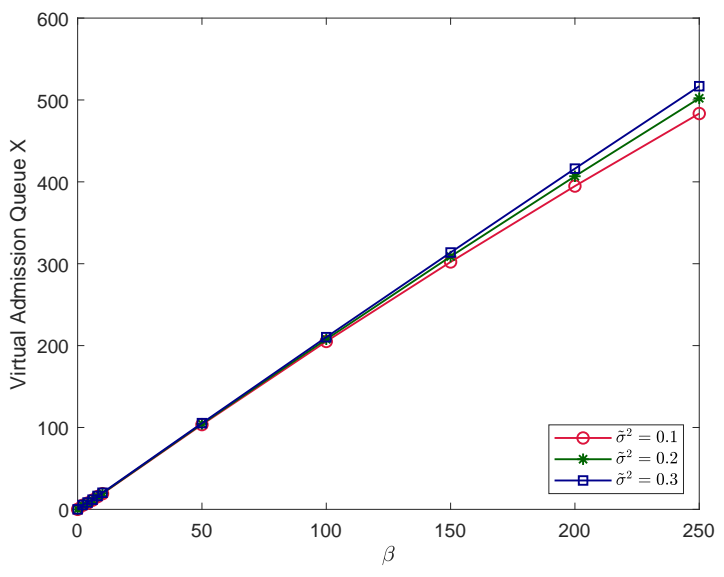

Fig. 5. Time-averaged virtual admission queue versus $\beta$.

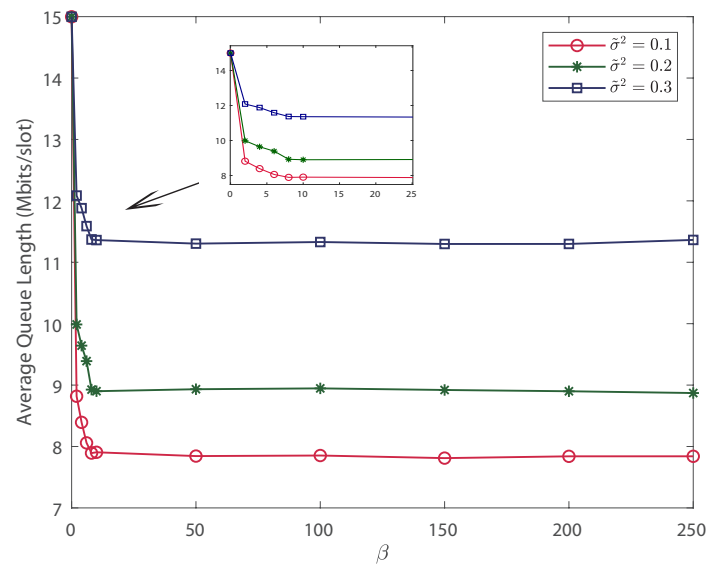

Fig. 6. Time-averaged data queue length against $\beta$.

imized at each time slot, and this validates the conclusion of Theorem 2(c). From the curves, with a large value of the CSI estimation error $\tilde{\alpha}^{2}$, the stabilized time-averaged system throughput becomes bad. This is because the transmission rate is a decreasing function with the estimation error variance $\tilde{\alpha}^{2}$. Thus, a large value of $\tilde{\alpha}^{2}$ leads to a small transmission rate, and then a low system throughput.

Fig. 5 illustrates the time-averaged virtual queue backlog $X_{m}(t)$ with respect to different values of $\beta$. It can be seen that the time-averaged virtual queue length increases as $\beta$ increases, and this validates the conclusion of Theorem $2(b)$. This is because a larger value of $\beta$ means a higher priority to maximize the system throughput, and thus leads to a higher admission rate.

Fig. 6 shows the time-averaged data queue backlog of $Q_{m}(t)$ by varying control parameter $\beta$. We can see that the average queue backlog decreases when $\beta<10$, and then slows down the decrease trend and starts to stabilize when $\beta>10$. We observe that a large value of the estimation error $\tilde{\alpha}^{2}$ leads to a large data queue backlog. This is because a large value of $\tilde{\alpha}^{2}$ leads to a small transmission rate, and thus a large data queue length. 

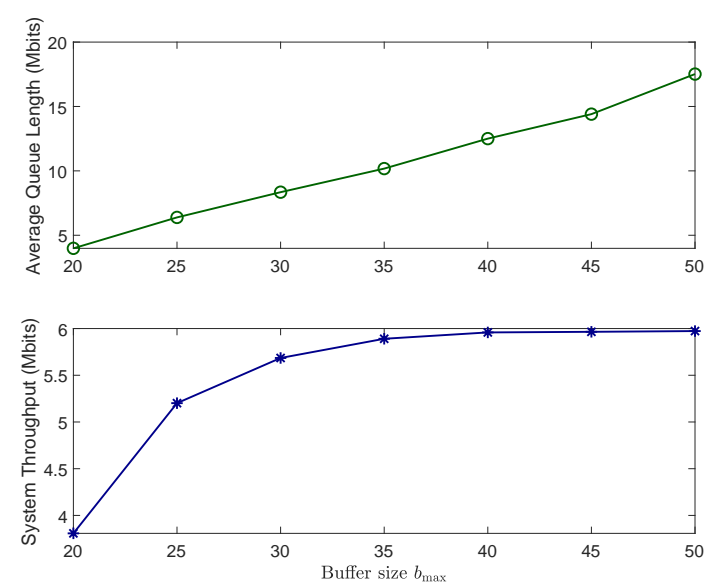

Fig. 7. Network performance versus $b_{\max }$.

Fig. 7 plots the performance of TORS with the variation of $b_{\max }$ with $\beta=100$. We observe that a large buffer size can improve the network performance of IoT systems. With a larger value of $b_{\max }$, the system allows more sensory data into the data queue at IoT devices for transmission and thus the time-averaged queue length is increased.

\section{CONCLUSION}

In this paper, we have studied the resource scheduling to maximize the time-averaged system throughput for OFDMAbased uplink IoT systems. A stochastic optimization problem has been formulated and solved through the exploitation of Lyapunov optimization theory and Lagrangian dual decomposition technique. An algorithm, referred to TORS, has been designed, which can push the system throughput arbitrarily close to the optimal without any prior knowledge of CSI and task arrival information.

\section{ACKNOWLEDGEMENTS}

This work is supported in part by the National Key Research and Development Program under Grant no. 2016YFB1000102, in part by the National Natural Science Foundation of China under Grant no. 61972222, 61672318, 61631013, and in part by the projects of Tsinghua National Laboratory for Information Science and Technology (TNList), supported by Academician Expert Workstation of Bitvalue Technology (Hunan) Company Limited (2017RS3064).

\section{APPENDIX A}

\section{Proof OF THEOREM 1}

Lemma 1: For any nonnegative real numbers $x, y$ and $z$, there holds $[\max (x-y, 0)+z]^{2} \leq x^{2}+y^{2}+z^{2}+2 x(z-y)$ [8].
According to Lemma 1, we obtain

$$
\begin{aligned}
& \frac{1}{2 b_{\max }}\left[X_{m}(t+1) Q_{m}(t+1)^{2}-X_{m}(t) Q_{m}(t)^{2}\right] \\
& \leq \frac{1}{2 b_{\max }}\left[\left(X_{m}(t)+V_{m}(t)\right) Q_{m}(t+1)^{2}-X_{m}(t) Q_{m}(t)^{2}\right] \\
& \leq \frac{1}{2 b_{\max }}\left[V_{m}(t) Q_{m}(t+1)^{2}\right] \\
& +\frac{X_{m}(t)}{2 b_{\max }}\left[C_{m}(t)^{2}+R_{m}(t)^{2}+2 Q_{m}(t)\left(R_{m}(t)-C_{m}(t)\right)\right] \\
& \leq \frac{A_{\max } b_{\max }}{2}+\frac{C_{m}(t)^{2}+R_{m}(t)^{2}}{2 b_{\max }} X_{m}(t) \\
& +\frac{R_{m}(t)-C_{m}(t)}{b_{\max }} Q_{m}(t) X_{m}(t)
\end{aligned}
$$

By squaring both sides of (8), and employing (35), we obtain

$$
\begin{aligned}
& \Delta(\boldsymbol{\Theta}(t))=\mathbb{E}\{L(\boldsymbol{\Theta}(t+1))-L(\boldsymbol{\Theta}(t)) \mid \boldsymbol{\Theta}(t)\} \\
& \leq B+\sum_{m=1}^{M} \mathbb{E}\left\{\frac{C_{m}(t)^{2}+R_{m}(t)^{2}}{2 b_{\max }} X_{m}(t) \mid \boldsymbol{\Theta}(t)\right\} \\
& -\sum_{m=1}^{M} \mathbb{E}\left\{\frac{X_{m}(t) Q_{m}(t)}{b_{\max }} C_{m}(t) \mid \boldsymbol{\Theta}(t)\right\} \\
& +\sum_{m=1}^{M} \mathbb{E}\left\{R_{m}(t) \frac{X_{m}(t)}{b_{\max }}\left[Q_{m}(t)-\left(b_{\max }-A_{\max }\right)\right] \mid \boldsymbol{\Theta}(t)\right\} \\
& +\sum_{m=1}^{M} \mathbb{E}\left\{\frac{b_{\max }-A_{\max }}{b_{\max }} X_{m}(t) V_{m}(t) \mid \Theta(t)\right\}
\end{aligned}
$$

where

$$
\begin{aligned}
B \geq B(t)= & \frac{b_{\max }-A_{\max }}{2 b_{\max }} \sum_{m=1}^{M}\left(R_{m}(t)^{2}+V_{m}(t)^{2}\right) \\
& +\frac{M A_{\max } b_{\max }}{2}
\end{aligned}
$$

Subtracting $\beta \mathbb{E}\left\{V_{\text {sum }}(t) \mid \Theta(t)\right\}$ on both sides of (36), we can prove the Theorem 1 .

\section{APPENDIX B}

\section{PROOF OF THEOREM 2}

Lemma 2: For any feasible rate vector $\pi \in \Omega$ which denotes the capacity region, there exists a randomized stationary policy that chooses the admission control decision and resource scheduling decision in each time slot $t$ independent of queue backlog, and there exists the following steady-state equations

$$
\mathbb{E}\left\{C_{m}(t)\right\}=\mathbb{E}\left\{R_{m}(t)\right\}=\mathbb{E}\left\{V_{m}(t)\right\}=\pi_{m}
$$

The proof of Lemma 2 can be found in [28], and we omit the detail here for brevity.

Let $\mathbb{E}\left\{R_{m}^{*}(t)\right\}=r_{m}^{*}$. Note that $\pi_{m}$ can take values as $r_{m, \epsilon}^{*}$ or $r_{m, 2 \epsilon}^{*}$, where $\epsilon$ is a positive, arbitrarily small number. The admission rate can be controlled ranging from $r_{m}^{*}$ to $r_{m, \epsilon}^{*}$ or to $r_{m, 2 \epsilon}^{*}$. Both of them are within $\Omega$. 
According to the Theorem 1, we have

$$
\begin{aligned}
& \Delta(\boldsymbol{\Theta}(t))-\beta \mathbb{E}\left\{V_{\text {sum }}(t) \mid \boldsymbol{\Theta}(t)\right\} \\
& \leq B+\sum_{m=1}^{M} \mathbb{E}\left\{\frac{C_{m}(t)^{2}+R_{m}(t)^{2}}{2 b_{\max }} X_{m}(t) \mid \boldsymbol{\Theta}(t)\right\} \\
& -\sum_{m=1}^{M} \mathbb{E}\left\{\frac{X_{m}(t) Q_{m}(t)}{b_{\max }} C_{m}(t) \mid \Theta(t)\right\} \\
& +\sum_{m=1}^{M} \mathbb{E}\left\{R_{m}(t) \frac{X_{m}(t)}{b_{\max }}\left[Q_{m}(t)-\left(b_{\max }-A_{\max }\right)\right] \mid \boldsymbol{\Theta}(t)\right\} \\
& +\sum_{m=1}^{M} \mathbb{E}\left\{V_{m}(t)\left(\frac{b_{\max }-A_{\max }}{b_{\max }} X_{m}(t)-\beta\right) \mid \Theta(t)\right\}
\end{aligned}
$$

The third, fourth, and fifth terms on the RHS of (39) are minimized by the resource scheduling, admission control, admitted admission control, respectively. Substituting admission rate vector $r_{m, 2 \epsilon}^{*}$ into the third and fourth terms, and substituting admitted admission rate vector $r_{m, \epsilon}^{*}$ into the fifth term, and employing (38), we have

$$
\begin{aligned}
& \Delta(\boldsymbol{\Theta}(t))-\beta \mathbb{E}\left\{V_{\text {sum }}(t) \mid \boldsymbol{\Theta}(t)\right\} \\
& \leq B+\sum_{m=1}^{M} \frac{X_{m}(t)}{b_{\max }}\left[\frac{C_{m}(t)^{2}+R_{m}(t)^{2}}{2}-\left(b_{\max }-A_{\max }\right) \epsilon\right] \\
& \quad-\beta \sum_{m=1}^{M} r_{m, \epsilon}^{*}
\end{aligned}
$$

For $\vartheta>0$, such that $\vartheta \leq\left(2 \epsilon\left(b_{\max }-A_{\max }\right)-\left(C_{m}(t)^{2}+\right.\right.$ $\left.\left.R_{m}(t)^{2}\right)\right) / 2 b_{\max }$. We obtain

$$
\begin{aligned}
& \Delta(\boldsymbol{\Theta}(t))-\beta \mathbb{E}\left\{V_{\text {sum }}(t) \mid \boldsymbol{\Theta}(t)\right\} \\
& \leq B-\vartheta \sum_{m=1}^{M} X_{m}(t)-\beta \sum_{m=1}^{M} r_{m, \epsilon}^{*}
\end{aligned}
$$

Taking expectations on both sides of (41) and summing up all the telescoping over $\{0,1, \ldots, T-1\}$, we can obtain

$$
\begin{aligned}
& \mathbb{E}\{L(\boldsymbol{\Theta}(T))\}-\mathbb{E}\{L(\boldsymbol{\Theta}(0))\}-\beta \sum_{t=0}^{T-1} \mathbb{E}\left\{V_{\text {sum }}(t)\right\} \\
& \leq T B-\vartheta \sum_{t=0}^{T-1} \sum_{m=1}^{M} \mathbb{E}\left\{X_{m}(t)\right\}-\beta \sum_{t=0}^{T-1} \sum_{m=1}^{M} r_{m, \epsilon}^{*}
\end{aligned}
$$

Applying the definition of $L(\boldsymbol{\Theta}(t))$ and using the fact $L(\boldsymbol{\Theta}(T)) \geq 0$ and $L(\boldsymbol{\Theta}(0))=0$, and rearranging terms, we can simply have the inequality below

$$
\begin{aligned}
\vartheta \sum_{t=0}^{T-1} \sum_{m=1}^{M} \mathbb{E}\left\{X_{m}(t)\right\} \leq & T B+\beta \sum_{t=0}^{T-1} \mathbb{E}\left\{V_{\text {sum }}(t)\right\} \\
& -\beta \sum_{t=0}^{T-1} \sum_{m=1}^{M} r_{m, \epsilon}^{*} \\
\leq & T B+\beta T M A_{\max }
\end{aligned}
$$

Dividing by $T \vartheta$ and taking a limit as $T \rightarrow \infty$, we can prove Theorem 2(b). Thus, virtual queues $\boldsymbol{X}(t)$ is strongly stable. For $Q_{m}(t) \leq b_{\max }$ of Theorem 2(a), we can find the similar proof in [7] and omit the detail here for brevity.

Similarly, we can prove Theorem 2(c).

\section{REFERENCES}

[1] Y. Mao, C. You, J. Zhang, K. Huang, and K. B. Letaief, "A survey on mobile edge computing: the communication perspective," IEEE Commun. Surveys Tuts., vol. 19, no. 4, pp. 2322-2358, 2017.

[2] A. D. Dwivedi, P. Morawiecki, and G. Srivastava, "Differential cryptanalysis of round-reduced speck suitable for internet of things devices," IEEE Access, vol. 7, pp. 16476-16486, 2019.

[3] J. Sakhnini, H. Karimipour, A. Dehghantanha, R. M. Parizi, and G. Srivastava, "Security aspects of Internet of Things aided smart grids: a bibliometric survey," Internet of Things, pp. 100111, 2019.

[4] K. Wang, H. Yin, W. Quan, and G. Min, "Enabling collaborative edge computing for software defined vehicular networks," IEEE Network, vol. 32, no. 5, pp. 112-117, 2018.

[5] J. Ren, S. Yue, D. Zhang, Y. Zhang, and J. Cao, "Joint channel assignment and stochastic energy management for RF-powered OFDMA WSNs," IEEE Trans. Veh. Technol., vol. 68, no. 2, pp. 1578-1592, 2019.

[6] X. Cheng, Y. Wu, G. Min, and A. Y. Zomaya, "Network function virtualization in dynamic networks: a stochastic perspective," IEEE J. Sel. Areas Commun., vol. 36, no. 10, pp. 2218-2232, 2018.

[7] Y. Guo, Q. Yang, J. Liu, and K. S. Kwak, "Cross-layer rate control and resource allocation in spectrum-sharing OFDMA small-cell networks with delay constraints," IEEE Trans. Veh. Technol., vol. 66, no. 5, pp. 41334147, 2017.

[8] X. Xiang, C. Lin, X. Chen, and X. Shen, "Toward optimal admission control and resource allocation for LTE-A femtocell uplink," IEEE Trans. Veh. Technol., vol. 64, no. 7, pp. 3247-3261, 2015.

[9] X. Lyu, W. Ni, H. Tian, R. Liu, X. Wang, G. B. Giannakis, and A. Paulraj, "Optimal schedule of mobile edge computing for Internet of Things using partial information," IEEE J. Sel. Areas Commun., vol. 35, no. 11, pp. 2606-2615, 2017.

[10] H. Zhang, C. Jiang, N. C. Beaulieu, X. Chu, X. Wen, and M. Tao, "Resource allocation in spectrum-sharing OFDMA femtocells with heterogeneous services," IEEE Trans. Commun., vol. 62, no. 7, pp. 2366-2377, 2014.

[11] Y. Li, M. Sheng, C. Wang, X. Wang, Y. Shi, and J. Li, "Throughput-delay tradeoff in interference-free wireless networks with guaranteed energy efficiency," IEEE Trans. Wireless Commun., vol. 14, no. 3, pp. 1608-1621, 2015.

[12] S. Huang, B. Liang, and J. Li, "Distributed interference and delay aware design for D2D communication in cellular networks," in Proc. IEEE GLOBECOM, Washington, DC, USA, Dec. 2016, pp. 7-12.

[13] X. Song, L. Dong, J. Wang, L. Qin, and X. Han, "Energy efficient power allocation for downlink NOMA heterogeneous networks with imperfect CSI," IEEE Access, vol. 7, pp. 39329-39340, 2019.

[14] M. R. Zamani, M. Eslami, M. Khorramizadeh, and Z. Ding, "Energyefficient power allocation for NOMA with imperfect CSI," IEEE Trans. Veh. Technol., vol. 68, no. 1, pp. 1009-1013, 2019.

[15] J. Choi, N. Lee, S. N. Hong, and G. Caire, "Joint user selection, power allocation, and precoding design with imperfect CSIT for multi-cell MUMIMO downlink systems," CoRR, vol. abs/1902.00824, 2019. [Online]. Available: https://arxiv.org/abs/1902.00824.

[16] Z. Bai, M. Li, Y. Dong, H. Zhang, and P. Ma, "Joint fair resource allocation of D2D communication underlaying downlink cellular system with imperfect CSI," IEEE Access, vol. 6, pp. 63131-63142, 2018.

[17] X. Liu, J. Zhang, and S. Cai, "An optimal power allocation scheme in downlink multi-user NOMA beamforming system with imperfect CSI," in Proc. IEEE ICCS, Chengdu, China, Dec. 2018, pp. 99-103.

[18] F. Liu, Q. Yang, Q. He, D. Park, and K. S. Kwak, "Dynamic power and subcarrier allocation for downlink OFDMA systems under imperfect CSI," Wireless Networks, vol. 25, no. 2, pp. 545-558, 2019.

[19] L. Jiao, H. Yin, Y. Lyu, H. Huang, J. Dong, and D. Guo, "Optimal schedule of mobile edge computing under imperfect CSI," in Proc. IEEE ICA3PP, Guangzhou, China, Nov. 2018, pp. 32-45.

[20] S. B. Lee, I. Pefkianakis, A. Meyerson, S. Xu, and S. Lu, "Proportional fair frequency-domain packet scheduling for 3GPP LTE uplink," in Proc. IEEE INFOCOM, Rio de Janeiro, Brazil, Apr. 2009, pp. 2611-2615.

[21] S. Adireddy, L. Tong, and H. Viswanathan, "Optimal placement of training for frequency-selective block-fading channels," IEEE Trans. Inf. Theory, vol. 48, no. 8, pp. 2338-2353, 2002.

[22] F. Liu, Q. Yang, P. Gong, and K. S. Kwak, "Subcarrier and power allocation for multi-user OFDMA wireless networks under imperfect channel state information," IET Commucations, vol. 10, no. 8, pp. 873881, 2016.

[23] Y. Kim, J. Kwak, and S. Chong, "Dual-side optimization for cost-delay tradeoff in mobile edge computing," IEEE Trans. Veh. Technol., vol. 67, no. 2, pp. 1765-1781, 2018. 
[24] L. Pu, X. Chen, J. Xu, and X. Fu, "D2D fogging: an energy-efficient and incentive-aware task offloading framework via network-assisted D2D collaboration," IEEE J. Sel. Areas Commun., vol. 34, no. 12, pp. 3887 3901, Dec. 2016.

[25] M. J. Neely, "Stochastic network optimization with application to communication and queueing systems," Synthesis Lectures on Communication Networks, vol. 3, San Rafael, CA, USA: Morgan \& Claypool, 2010, pp. $1-211$.

[26] L. Georgiadis, M. J. Neely, and L. Tassiulas, "Resource allocation and cross-layer control in wireless networks," Foundations and Trends in Networking, vol. 1, no. 1, pp. 1-144, 2006.

[27] S. Boyd, and L. Vandenberghe, Convex optimization, Cambridge, U.K.: Cambridge Univ. Press, 2004

[28] M. J. Neely, "Energy optimal control for time varying wireless networks," IEEE Trans. Inf. Theory, vol. 52, no. 7, pp. 2915-2934, Jul. 2006.

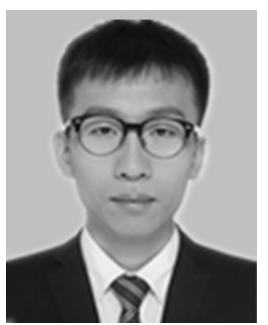

Libo Jiao received the B.S. degree from Wuhan University of Technology, Wuhan, China, in 2012, and the M.S. degree from Beihang University, Beijing, China, in 2015. He is currently pursuing the Ph.D. degree in Department of Computer Science at Tsinghua University, Beijing, China. His research interests include machine learning and stochastic optimization in wireless networks.

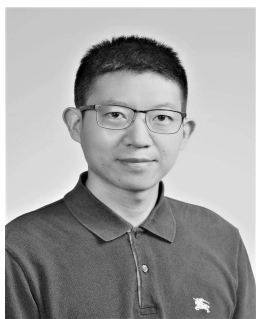

Yulei Wu is a Senior Lecture in the Department of Computer Science with the University of Exeter, United Kingdom. He received the Ph.D. degree in Computing and Mathematics and the B.Sc. degree (1st Class Hons.) in Computer Science from the University of Bradford, United Kingdom, in 2010 and 2006, respectively. His expertise is on networking and his main research interests include intelligent networking technologies, network slicing and softwarization, future Internet architecture and technologies, Green networking, wireless network, network security and privacy, and analytical modelling and performance optimization.

$\mathrm{He}$ is an Editor of IEEE Transactions on Network and Service Management, Elsevier Computer Networks, and IEEE Access. He contributes to major conference on networking as various roles including a Steering Committee Chair, a General Chair, a Program Chair, and a Technical Program Committee Member. His research has been supported by Engineering and Physical Sciences Research Council (EPSRC) of United Kingdom, National Natural Science Foundation of China, University's Innovation Platform and industry. $\mathrm{He}$ is a Senior Member of the IEEE, and a Fellow of the HEA (Higher Education Academy).

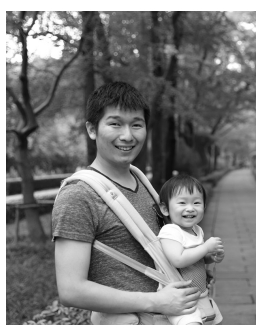

Jiaqing Dong received the B.S. degree in computer science from Peking University, Beijing, China, in 2013. He is currently pursuing the Ph.D. degree in Department of Computer Science at Tsinghua University, Beijing, China. His research interests include data center networks and distributed systems.
Zexun Jiang received the B.S. degree in computer science from Tsinghua University, Beijing, China, in 2014. He is currently pursuing the Ph.D. degree in Department of Computer Science at Tsinghua University. His research interests include network measurement and information retrieval. 